\title{
Improving Route Stability in Mobile Ad hoc Networks Based on Link Lifetime
}

\author{
Wenjing Yang \\ ${ }^{1}$ Dept. of Computer Science and Technology, Xi'an Jiaotong University, Xi'an, China \\ ${ }^{2}$ Xi' an Communication Institute, Xi'an, China \\ Email: xianywj@gmail.com \\ Xinyu Yang \\ Dept. of Computer Science and Technology, Xi'an Jiaotong University, Xi'an, China \\ Email: yxyphd@mail.xjtu.edu.cn \\ Shusen Yang \\ Dept. of Computing, Imperial College London, London, UK \\ Email: s.yang09@imperial.ac.uk \\ Yage Gong \\ Xi'an Communication Institute, Xi'an, China \\ Email: yaggong@gmail.com
}

\begin{abstract}
Mobile ad hoc networks (MANETs) have dynamic topology since all nodes have a random mobility behavior, which increases rerouting frequency and degrades communication performance. A more stable route can prolong route lifetime and reduce rerouting frequency. This paper proposes a Link lifetime based Backup Routing (LBR) protocol to improve route stability. LBR is based on link lifetime. It obtains the shortest path between source and destination through limited flooding as the primary path, and then sets up a local backup path for each link in the primary path concerning link lifetime. This scheme effectively avoids backup paths being out-of-date prematurely and increases the availability of backup paths. Both theoretical analyses and simulation results show that LBR has an improvement in route lifetime, packet delivery ratio, end-to-end delay, and so on, compared with singlepath routing protocols.
\end{abstract}

Index Terms—ad hoc networks, multipath routing, stability, backup routing, link lifetime

\section{INTRODUCTION}

In mobile Ad hoc networks (MANETs), the dynamic topology caused by node mobility leads to a poor route stability and thus has a negative impact on network performances.

The classical routing protocols are mostly single-path routing in which the path was constructed by Shortest Path Algorithm, such as DSR (Dynamic Source Routing) [1] and AODV (Ad hoc on demand distance vector Routing) [2]. In single-path routing protocols, when a route fails, the previous node of the failed link reports this failure to source; then source tries to construct a new

Manuscript received August 15, 2010; revised November 15, 2010; accepted January 15, 2011.

This work is supported by the China 863 Project (No.2006AA01Z210). route. However, such frequent route discoveries greatly increase control overhead, which on one hand consumes the limited wireless bandwidth intended for data transmissions and on the other hand consumes the finite energy of mobile nodes. Furthermore, for single-path routing without a suitable caching scheme, the corresponding data packets would be immediately dropped, which degrades the packet delivery ratio. Finally, frequent route discoveries also increase end-toend delay. Hence, to construct a stable route is an effective way to improve network communication performances in MANETs, especially those with requirements of high route stability, such as the disaster relief environment.

Route stability mainly relies on route lifetime [3], which is related to the route length and the lifetime of each link. That is to say a stable route should satisfy two conditions simultaneously: first, the path should be as short as possible, because the lifetime of route is approximately inversely proportional to the length of route [4]; second, link lifetime should be as long as possible, because the lifetime of route is the minimum lifetime of links in a route.

In large-scale MANETs, the route stability is very important. The hop count of a route may be large. If a route failed, the procedure of route rediscovery increases control overhead and end-to-end delay. We must construct a route with minimum hop counts and each link of the route with long lifetimes.

The stable single-path routing protocol HARP (Heading-direction Angles Routing Protocol) [5] prolongs route lifetime by extending link lifetime, but without any consideration on route length. Although some routing protocols construct a path with the least hop counts between source and destination, they do not achieve the longest link lifetime, e.g., GPSR (Greedy Perimeter Stateless Routing) [6]. Multipath backup 
routing is another way to improve route stability [7]. Several paths with low correlation are set up during route discovery. If the primary path fails, the data communication continues through a backup path; thus the route lifetime is prolonged and route rediscovery frequency is also reduced.

To improve route stability in large-scale MANETs, this paper presents a multipath routing protocol LBR (Link lifetime based Backup Routing) based on link lifetime. LBR sends RREQ (Route Request) in a limited flooding manner to discover a primary path between source and destination with the least hop counts, and twohop local backup paths are set up for each link in the primary path during the RREP (Route Reply) period. If the primary path fails, data transmission is continued through the corresponding local backup path. Local backup paths are constructed based on the condition that the lifetime of the local backup path is longer than lifetime of the corresponding link in the primary path, thus successfully avoiding backup path being out-of-date prematurely.

LBR has the following salient stable features: (i) In LBR, the primary path length is the shortest; (ii) Local backup path remains available when the primary path fails (a prediction method is introduced to achieve this function). Local backup path prolongs the link lifetime of primary path; and (iii) LBR greatly reduces the control overhead because the limited flooding mechanism is adopted in its route discovery procedure and local backup path can greatly reduce the rerouting frequency.

We use OPNET simulations to evaluate the effectiveness of LBR in comparison with a representative protocol, HARP [5]. Simulation results show that LBR achieves much better performance than HARP in terms of low overhead and high accuracy.

The rest of this paper is organized as follows. Section II discusses related research work on stable routing protocol. In Section III, we introduce the network model. In Section IV, we describe route discovery and maintenance in detail. In Section $\mathrm{V}$, we evaluate the performance of LBR protocol through theoretical analysis. In Section VI, the performance of LBR protocol is validated through OPNET simulations. Finally, we conclude the paper in Section VII.

\section{RELATED WORK}

In recent years, scholars have done great work on stable routing.

HARP (Heading-direction Angles Routing Protocol) [5] makes use of direction information of nodes in the network to reduce routing overhead and increase the links lifetime. Each link between two communicating nodes is selected based on the longest duration of existence.

LRP (Long-lived Route Prediction) scheme [8] predicts long-lived route based on the history of link lifetime. LRP does not use the GPS or the received signal strength which is affected by the presence of shadow effect. Each node periodically broadcasts a one-hop hello packet to monitor the lifetime of links of its neighbors and maintain a record of link lifetime.

SWORP (Stable Weight-based On-demand Routing Protocol) [9] uses the weight-based route strategy to select a stable route in order to enhance system performance. The weight of a route is decided by three factors: the route expiration time, the error count, and the hop count. Route discovery usually first discovers multiple routes from the source node to the destination node. Then the path with the largest weight is selected.

Many existing multipath routing protocols are mainly concerning issues caused by dynamic network topology, such as instability.

BSR (Backup Source Routing) [7] uses a heuristic cost function to achieve the lowest similarity between the backup path and the primary path to improve route stability. However, the source routing scheme is adopted in routing data, that is, the primary path and backup path information are included in the data packet, thus causing an extra overhead.

CHAMP (CacHing And Multiple Path Routing) [10] with data caching based on Ad-hoc On-demand Distance Vector (AODV) routing, sets up a relatively short backup path between source and destination. Since a longer path more easily leads to path failure, and the expected route lifetime is inversely proportional to the path length. However, it does not consider the link lifetime, the only criterion to choose the best path from source to destination is the hop count.

AODV-BR (Backup Route) [11] adopts an overhearing scheme to set up backup paths, while bringing no extra control overhead. However, for all these multipath protocols, no strict method is introduced to guarantee the availability of the backup path in case of primary path failure, i.e., the backup path outdatedness.

AODV-ABR (Adaptive Backup Routing for ad-hoc networks) [12] adopts both RREP and data packets overhearing to set up backup paths. Although it obtains the latest backup path information to avoid the backup path outdated, all nodes are in the promiscuous mode, which greatly consumes the scarce energy resource.

\section{THE NETWORK MODEL}

We assume all nodes are identical and follow an independent uniform distribution in a two-dimensional space. Each node has a unique wireless channel with the same transmission range $R$ and has an omnidirectional antenna; the communication link is bi-directional. Two nodes are supposed to be connected when the distance of them is less than $R$. Each node knows its own location information through GPS (Global Position System), and can obtain other nodes' location via a location service protocol such as GLS [13].

We assume that each node knows exactly its own location and the destination's location.

Based on the above assumptions, the network can be considered as a graph $G=(V, E)$, where $V$ is the set of all nodes and $E$ is the set of all edges. The path between 
source $v_{S}$ and destination $v_{D}$ can be represented as a node sequence $\mathcal{P}=\left(v_{S}, \ldots, v_{i}, \ldots, v_{D}\right)$, where $v_{i} \in V$.

In this paper the shortest path discovered by using limited flooding is defined as the primary path between $v_{S}$ and $v_{D}$, denoted by $\mathcal{P}_{\text {primaty }}=\left(v_{S}, v_{1}, \ldots, v_{D}\right)$. Node $v_{i}$ 's neighbor node set is denoted as $\mathcal{S}_{i}$, and the common node set of $v_{i-1}$ and $v_{i}$ is $\mathcal{C}_{i}=\mathcal{S}_{i-1} \cap \mathcal{S}_{i}$.

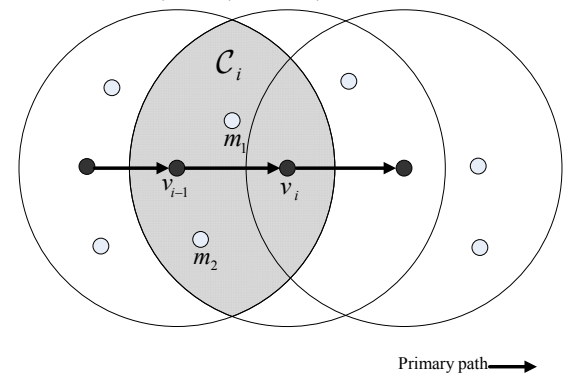

Figure 1. An example of common node set $\mathcal{C}_{i}$.

As shown in Fig. 1, $v_{i-1}$ and $v_{i}$ are two neighboring nodes in the primary path, and then the common node set is $\mathcal{C}_{i}=\left\{m_{1}, m_{2}\right\}$.

Denote $\operatorname{LET}\left(v_{i-1}, v_{i}\right)$ and $\operatorname{PET}(\mathcal{P})$ as lifetimes of link $\left(v_{i-}\right.$ $\left.{ }_{1}, v_{i}\right)$ and path $\mathcal{P}$ respectively. For two given neighboring nodes $v_{i-1}$ and $v_{i}$ in the primary path, assume there is a common node $u_{j}$, it satisfies:

$$
\begin{aligned}
& u_{j} \in \mathcal{C}_{i} \wedge \operatorname{PET}\left(v_{i-1}, u_{j}, v_{i}\right)>\operatorname{LET}\left(v_{i-1}, v_{i}\right), \\
& \wedge \forall u_{k} \in \mathcal{C}_{i}, \operatorname{PET}\left(v_{i-1}, u_{j}, v_{i}\right)>\operatorname{PET}\left(v_{i-1}, u_{k}, v_{i}\right)
\end{aligned}
$$

then there exists a local backup path $\operatorname{Path}_{\text {backup }}^{i-1, i}=\left(v_{i-1}, u_{j}, v_{i}\right)$ for the two neighboring nodes $v_{i-1}$ and $v_{i}$ in the primary path. Denote the set of all local backup paths of the primary path by $\mathcal{P}_{\text {backup }}=\left\{\right.$ Path $\left._{\text {backup }}^{i-1, i} \mid\left(v_{i-1}, v_{i}\right) \in \mathcal{P}_{\text {primary }}\right\}$. Finally, backup route is defined as $\left(\mathcal{P}_{\text {primaty }}, \mathcal{P}_{\text {backup }}\right)$, including the primary path and all the local backup paths for the links in the primary path.

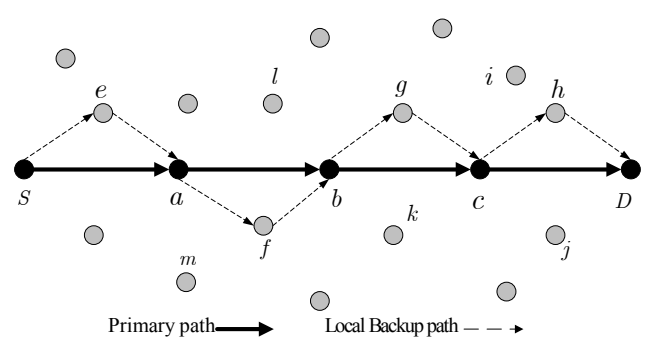

Figure 2. Primary path and local backup paths of LBR.

For example, as shown in Fig. 2, the primary path between source $S$ and destination $D$ is $(S, a, b, c, D)$, and the local backup path for link $(a, b)$ is $(a, f, b)$.

\section{LBR PROTOCOL}

In this section, we first overview LBR protocol and then present the detailed components of LBR.

\section{A. Overview}

LBR is an on-demand multipath stable routing protocol, the primary path with the minimum hop counts between source and destination, and the local backup path has longer lifetime than corresponding link in the primary path.

When a source attempts to send a packet to its destination, it checks its routing table first. If there is not an entry for the destination, the route discovery process starts. The route discovery procedure starts with the source broadcasting a RREQ. The RREQ is flooded in a request zone that is determined by the locations of source and destination. Duplicated RREQ is dropped to reduce flooding overhead.

When the destination receives several RREQs, it chooses the path that has the least hop counts as the primary path. And then it replies with a RREP along the reverse path; then the intermediate nodes construct local backup paths in a contending manner, and record the reverse path to source and the forward path to destination.

The route discovery procedure is complete when the source receives the RREP. Data packets are initially transmitted through the primary path. However when the primary path fails, a local backup path is adopted to continue data transmission. Once the corresponding local backup path fails at the same time, the data packets are dropped and a RERR (Route Error) is sent back to source. Through this mechanism, LBR prolongs route lifetime, and improves the route stability.

\section{B. Route Discovery}

Let $S$ and $D$ denote the source and the destination respectively. Route discovery is started before transmitting a data packet if $S$ can not find a route to $D$.

1) The preparation of Route Discovety

During the route discovery, LBR uses location information of nodes to flood a RREQ packet for destination in a request zone instead of in the entire network. This mechanism is the same as Location-aided routing (LAR) [14]. This request zone is defined by location information on source and destination.

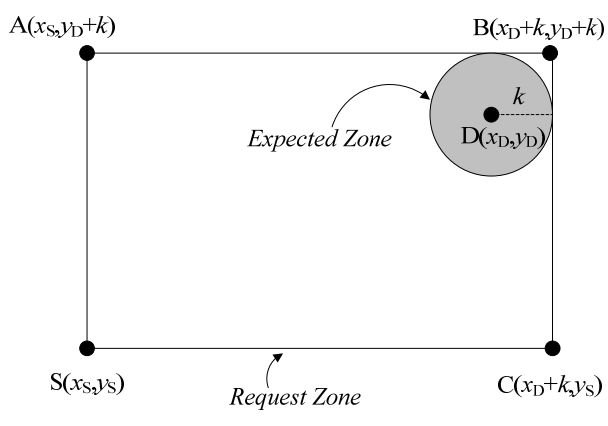

Figure 3. Expected zone and request zone.

In Fig. 3, source $S$ floods RREQ in the request zone by using the location of $S$ and expected zone for destination node $D$. The expected zone is a circular area around the location of $D\left(D\right.$ 's coordinate is $\left.\left(x_{D}, y_{D}\right)\right)$, and its radius $k$ 
depends on the average velocity $V_{a v g}$ of the mobile node and on the time elapsed since the last known location of the destination was recorded.

$$
k=V_{\text {avg }} * \Delta t
$$

here $\Delta t$ is the duration between two successive route requests from the source node.

In Fig. 3, the request zone is a rectangle whose corners are $S, A, B$, and $C$. Node $S$ 's coordinate is denoted by $\left(x_{S}, y_{S}\right)$, then we can get that the coordinates of nodes $A, B$, and $C$ are $\left(x_{S}, y_{D}+k\right), \quad\left(x_{D}+k, y_{D}+k\right)$, and $\left(x_{D}+k, y_{S}\right)$, respectively.

RREQ includes the four corners' coordinates. When a node receives the RREQ, it determines whether its location is in the request zone. If a node isn't in the request zone, it doesn't forward the RREQ. Flooding RREQ in the request zone greatly reduces the control overhead.

\section{2) Route Request Propagation}

In order to construct a primary path to destination, $S$ floods a RREQ in the request zone. RREQ has a structure of $[\pi, S, D, R I D, t t l, R Z]$, where $\pi=(S, \ldots, p)$ is the path traversed from $S$ to the current node $p, D$ is the destination, $R I D$ is route request packet ID to distinguish between different RREQs, and $t t l$ (time-to-live) is used for limited flooding, that is, every time RREQ is forwarded, $t t l$ is decremented by one, and RREQ is dropped if it has not reached $D$ even when $t t l$ counts down to 0 , which avoids unnecessary use of network resources. $R Z$ includes the coordinates of four corners of the request zone.

Each node maintains a route request list, which stores the latest received RREQ. We denote by $R I D_{i}(S)$, which means $R I D$ for source $S$, and is recorded in node $N_{i}$ 's route request list.

Algorithm 1. The procedure of primary path discovery:

Step 1) When the node $N_{i}$ who receives a RREQ is the destination $D$, stop;

Step 2) Else If the location of $N_{i}$ is not in the request zone, stop;

Step 3) Else if $N_{i}$ is already in the path $\pi$ recorded in the RREQ, then this RREQ is dropped and algorithm stops;

Step 4) Else if RREQ is in the node's route request list, then this RREQ is dropped and algorithm stops;

Step 5) The $t t l$ is decremented by 1 . If $t t l \leq 0$, stop;

Step 6) The current node $N_{i}$ adds its own ID into the path $\pi$, and then further broadcasts this RREQ $\left[\left(S, \ldots, p, N_{i}\right), S, D, R I D, t t l-1\right]$.

3) Route Reply Propagation

When several RREQs reach destination $D, D$ selects the path which has the least hop counts as the primary path, that is, the primary path is the path with the shortest length. Hence, LBR meets the first condition of stable route (in Section 1).

RREP is sent along the reverse path recorded in RREQ, and it includes the location and velocity information of node. Once all the intermediate nodes in the primary path set up a reverse path to source and a forward path to destination, the local backup paths are constructed for each link in the primary at the same time.

Local backup path has a longer lifetime than that of the corresponding link in the primary path. The lifetime of a path is restrained by the lifetime of each link in the path.

Meanwhile, lifetime of the link $\left(v_{i-1}, v_{i}\right)$ is calculated by

$$
\operatorname{LET}\left(v_{i-1}, v_{i}\right)=\frac{-(p l+q d)+\sqrt{\left(p^{2}+q^{2}\right) R^{2}-(p d-l q)^{2}}}{p^{2}+q^{2}},
$$

where $p=\tau_{i-1} \sin \theta_{i-1}-\tau_{i} \sin \theta_{i}, q=\tau_{i-1} \cos \theta_{i-1}-\tau_{i} \cos \theta_{i}, l=x_{i-1}-x_{i}$, $d=y_{i-1}-y_{i}$. $\left(x_{i-1}, y_{i-1}\right)$ and $\left(x_{i}, y_{i}\right)$ are the node coordinates, $\tau_{i-1}$ and $\tau_{i}$ are the node velocities, $\theta_{i-1}$ and $\theta_{i}$ are the direction angles, and $R$ is the transmission range [15].

$\forall m_{j} \in \mathcal{C}_{i}$, lifetime of the path $\left(n_{i-1}, m_{j}, n_{i}\right)$ is calculated by

$$
\operatorname{PET}\left(n_{i-1}, m_{j}, n_{i}\right)=\min \left(\operatorname{LET}\left(n_{i-1}, m_{j}\right), \operatorname{LET}\left(n_{i}, m_{j}\right)\right) \text {. }
$$

Now denote $N_{i}$ as the node that broadcasts RREP, $N_{i-1}$ as the last-hop node in the reverse path, $N_{i+1}$ as the nexthop node in the reverse path, and $M$ as a neighbor of $N_{i}$. When $M$ receives a RREP, it operates as algorithm 2 .

Algorithm 2. The RREP procedure:

Step 1) When node $M$ receives a RREP, it adds RREP information to its RREP list;

Step 2) If $M \neq N_{i+1}$ and $M \neq N_{i-1}$, go to Algorithm 3 to start local backup path setup;

Step 3) If $M=N_{i-1}$, take a record of $\operatorname{LET}\left(N_{i-1}, N_{i}\right)$ in this RREP;

Step 4) If $M=N_{i+1}$, calculate the link lifetime $\operatorname{LET}\left(N_{i}, N_{i+1}\right)$, as in (1); record the route information in the route list, and if $N_{i+1}$ isn't the source, then adds the next-hop IP into this RREP and broadcast it;

Step 5) If $M$ is the source, stop.

Algorithm 3. The procedure of local backup path discovery:

Step 1) If the intermediate node $M \neq N_{i+1}$ and $M \neq N_{i-1}$, and $M$ has received the same RREP before, it calculates the path lifetime $\operatorname{PET}\left(N_{\mathrm{i}-1}, M, N_{i}\right)$, see (2);

Step 2) $M$ broadcasts a contending packet $\mathrm{CB}$ after a delay of $d=\alpha / \operatorname{PET}\left(N_{i-1}, M, N_{i}\right)+\beta(\alpha$ and $\beta$ are scaling factors), and then records the path information to $N_{i-1}$ and $N_{i} ; M$ is blocked when it is hearing $\mathrm{CB}$ from other nodes;

Step 3) When $N_{i-1}$ and $N_{i}$ receive a CB packet, if $\operatorname{PET}\left(N_{i-}\right.$ $\left.{ }_{1}, M, N_{i}\right)<\operatorname{LET}\left(N_{i-1}, N_{i}\right)$, the corresponding path information is ignored; or else it is stored into the backup route list as a local backup path for link $\left(N_{i-1}, N_{i}\right)$.

We take Fig. 2 as an example for LBR route discovery. When $S$ is about to send data to $D$ but no route for $D$ is available, then route discovery is started.

In request zone, $S$ floods RREQ to construct a primary path $(S, a, b, c, D)$ with least hop counts. $D$ replies a RREP to $S$. The RREP includes the entire primary path and is forwarded by one-hop broadcasting. 
$D$ selects node $c$ as the next hop and sends RREP (we denote the RREP packet sent by $D$ as $R R E P_{D}$ ) to it. Here, the link $(D, c)$ of primary path is established. Nodes $h, i$, and $j$ may receive this RREP simultaneously. When $c$ receives $R R E P_{D}$, it records $R R E P_{D}$ in its RREP table and updates the corresponding fields of RREP $\left(R R E P_{D}\right)$ with its ID, location, velocity, and $\operatorname{LET}(D, c)$. Then, node $c$ selects $b$ as its next hop and sends $R R E P_{c}$ to $b\left(R R E P_{c}\right.$ denotes the RREP that has been updated by $c$ ).

When $h, i$, and $j$ receive $R R E P_{D}$, they add it to their respective RREP tables. When $h$ and $j$ receive the same $R R E P_{D}$, they calculate $\operatorname{PET}(D, h, c)$ and $\operatorname{PET}(D, j, c)$, respectively, based on Equation (2), and then broadcast CB packets after delays of $d_{h}=\alpha / \operatorname{PET}(D, h, c)+\beta$ and $d_{j}=\alpha / \operatorname{PET}(D, j, c)+\beta \quad, \quad$ respectively, if $\operatorname{PET}(D, h, c)>\operatorname{LET}(D, c)$ and $\operatorname{PET}(D, j, c)>\operatorname{LET}(D, c)$. Assume that $d_{h}$ is the node with the smallest delay; then $h$ broadcasts a CB packet, and $j$ is blocked by $h$ 's CB. After this procedure, the local backup path $(D, h, c)$ is established for the link $(D, c)$.

During RREP delivery, the links of the primary path and their local backup paths are established by the same operations. The primary path is constructed when RREQ is sent back to $D$, and the construction of the local backup path is completed when RREP reaches $S$.

\section{Route Maintenance}

Due to the dynamic topology caused by node mobility and the limited radio range, route failure becomes inevitable in MANETs, and thus a route maintenance mechanism is needed for LBR.

In LBR, nodes rely on data packet acknowledgment provided by the link layer to determine the state of a link. A link is declared as failed when the corresponding node has not received any acknowledgment from the next-hop after forwarding a data packet.

Since failure of any link in the primary path leads to route failure, LBR requires monitoring the availability of each link.

Algorithm 4. The procedure of Route maintenance:

Step 1) If a link in the primary path fails, the data packet is continued through the corresponding local backup path and the failed primary path is deleted at the same time. Before transmission, a flag is set in the data packet header to indicate the next-hop node in the corresponding local backup path that this data should be transmitted through the local backup path to the destination;

Step 2) On receiving a data packet with the flag set, this packet is unicasted to the next-hop node indicated by the local backup path when there exists a corresponding route entry in the local backup path list;

Step 3) When a node in the primary path receives a data packet, it then firstly forwards the packet through the primary route;
Step 4) Data is dropped when the corresponding local backup path also fails. And then RERR is sent back along the reverse path to inform the source and intermediate nodes of this failure;

Step 5) When an intermediate node receives RERR, the corresponding route in the node's is deleted.

Step 6) When the source receives RERR, it restarts a route discovery process.

Take Fig. 4 as an example for LBR route maintenance.

In Fig. 4, data packets are originally transmitted along the primary path $(S, a, b, c, D)$. When $c$ moves out of $b$ 's transmission range and $b$ detects the link failure, it deletes the corresponding path related to $c$ and selects a local backup path to the next node in the local backup path list. Then it sets a flag in the data packet and sends this packet to the next-hop node $e$ in the local backup path. On receiving this data packet, $e$ checks this flag and then forwards this packet through the local backup path directly to $c$. When $c$ receives this data packet through the corresponding local backup path, it uses the primary path to transmit this data to $D$ once again. If the local backup path from $b$ to $c$ also fails, then $b$ sends RERR to inform $S$ in order to start a new route discovery.

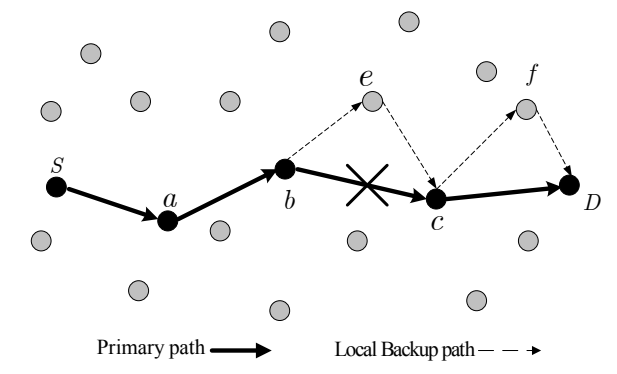

Figure 4. An example of LBR Route Maintenance.

\section{ANLYSIS ON LBR}

In this section, the analysis on LBR is divided into two sub-sections:

As route lifetime can reflect the stability of a routing protocol, we derive the CDF (Cumulative Distribution Functions) of the LBR's route lifetime in this section.

During the local backup path setting up process, the common nodes send CB packets in contention mode. Too many $\mathrm{CB}$ packets will lead to heavy control overhead and communication interference. Therefore, in this section, we derive the maximum number of nodes that are sending CB packets in a common set.

\section{A. Analysis on The Lifetime of $L B R$}

In this section, the lifetime of LBR is analyzed by mathematic method.

We use a random variable to represent lifetime of a link between two communicating nodes. Assume the route from $S$ to $D$ comprises $k$ links. Denote the $i$ th link as $L_{i}$ and its corresponding lifetime as $X_{L_{i}}$. Suppose $X_{L_{i}}$ $(i=1,2,3 \ldots, k)$ is an independently and identically distributed random variable, satisfying an exponential 
distribution with an expectation of $m$. Denote $T$ as route lifetime, then

$$
T=\min \left(X_{L_{1}}, X_{L_{2}}, \ldots, X_{L_{k}}\right) .
$$

$F_{X_{L i}}(t)$ is the Cumulative Distribution Function (CDF) of link $L_{i}$, and $t$ is link available time; then T's CDF is as follow:

$$
\begin{aligned}
F_{T}(t) & =P[T \leq t] \\
& =P\left[\min \left(X_{L_{1}}, X_{L_{2}}, X_{L_{3}}, \ldots X_{L_{k}}\right) \leq t\right] \\
& =P\left[\left(X_{L_{1}} \leq t\right) \cap\left(X_{L_{2}} \leq t\right) \cap\left(X_{L_{3}} \leq t\right) \cap \ldots \cap\left(X_{L_{k}} \leq t\right)\right] . \\
& =P\left(X_{L_{1}} \leq t\right) \cdot P\left(X_{L_{2}} \leq t\right) \cdot P\left(X_{L_{3}} \leq t\right) \ldots P\left(X_{L_{k}} \leq t\right) \\
& =1-\prod_{i=1}^{k}\left[1-F_{X_{L}}(t)\right]
\end{aligned}
$$

LBR routing model is shown as in Fig. 2. For every link in the primary path, there is a local backup path. Denote $X_{M_{i}}$ as lifetime of the $i$ th primary link, and $X_{B_{i}}$ as lifetime of the $i$ th local backup path, then lifetime of the $i$ th hop of the primary path is:

$$
X_{L_{i}}=\max \left(X_{M_{i}}, X_{B_{i}}\right) .
$$

The CDF of link $L_{i}$ is:

$$
\begin{aligned}
F_{L i}(t) & =P\left[X_{L_{i}} \leq t\right] \\
& =P\left[\max \left(X_{M_{i}}, X_{B_{i}}\right) \leq t\right] \\
& =F_{X_{M_{i}}}(t) \cdot F_{X_{B_{i}}}(t) \\
& =1-e^{-2 \lambda_{i} t}-e^{-\lambda_{i} t}+e^{-3 \lambda_{i} t}
\end{aligned}
$$

Through the combination of (4) and (6), it is easy to obtain that:

$$
\begin{aligned}
F_{T}(t)= & 1-\left(e^{-2 \lambda_{1} t}+e^{-\lambda_{1} t}-e^{-3 \lambda_{1} t}\right) \cdot\left(e^{-2 \lambda_{2} t}+e^{-\lambda_{2} t}-e^{-3 \lambda_{2} t}\right) \\
& \cdot \ldots \cdot\left(e^{-2 \lambda_{k} t}+e^{-\lambda_{k} t}-e^{-3 \lambda_{k} t}\right) \\
= & 1-\prod_{i=1}^{k}\left(e^{-2 \lambda_{i} t}+e^{-\lambda_{i} t}-e^{-3 \lambda_{i} t}\right) \\
= & 1-\left(e^{-2 \lambda t}+e^{-\lambda t}-e^{-3 \lambda t}\right)^{k}
\end{aligned}
$$

Perform a differentiation on (7), then $T$ 's Probability Density Function (PDF) is obtained as follow:

$$
f_{T}(t)=k\left(e^{-2 \lambda t}+e^{-\lambda t}-e^{-3 \lambda t}\right)^{k-1}\left(3 \lambda e^{-3 \lambda t}-2 \lambda e^{-2 \lambda t}-\lambda e^{-\lambda t}\right) .
$$

Since route length $k$ is already known, $T$ 's expectation $\mathrm{E}[T]$ is obtained from (8), E.g., when $k=3$, the expected $T$ is

$$
\begin{aligned}
E[T] & =\int_{-\infty}^{+\infty} t f_{T}(t) d t \\
& =\int_{-\infty}^{+\infty} 3 t\left(e^{-2 \lambda t}+e^{-\lambda t}-e^{-3 \lambda t}\right)^{2}\left(3 \lambda e^{-3 \lambda t}-2 \lambda e^{-2 \lambda t}-\lambda e^{-\lambda t}\right) d t \\
& =\frac{296}{576} m .
\end{aligned}
$$

For a single-path route with a length of 3 , the expected $T[16]$ is $E[T]=\frac{m}{3}$.

Through a comparison of the above two results, LBR improves route lifetime by $18 \%$ compared with singlepath routing protocol.

\section{B. Analysis on The Number of CB Packets}

During backup path setup for each link in the primary path, the common nodes broadcast $\mathrm{CB}$ packets in a competition manner. If too many $\mathrm{CB}$ packets are broadcast, backup path setup will be influenced by communication competition. Hence the number of common neighbor nodes who are sending $\mathrm{CB}$ packets should be as small as possible.

Theorem 1. During the path backup for each link in the primary path, the number of nodes (which are in the common area of two neighbor nodes in the primary path) located in $\mathcal{C}_{i}$ and sending $\mathrm{CB}$ packets is smaller than 4.

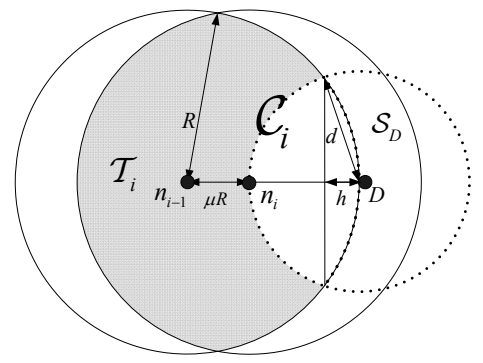

Figure 5. The common node set and the destination.

Proof. As shown in Fig. 5, $n_{i-1}$ and $n_{i}$ are two neighbor nodes located in the primary path, and $D$ is the destination node. The node transmission range is $R$, and we denote the distance between $n_{i}$ and $D$ as $d=\operatorname{Dis}\left(n_{i}, D\right)$. The distance between nodes $n_{i-1}$ and $n_{i}$ is $\mu R$, where $\operatorname{Dis}\left(n_{i-1}, n_{i}\right)=\mu R(0<\mu \leq 1) . \mathcal{C}_{i}$ is their common neighbor set. $\mathcal{S}_{D}$ denotes the circled area centered at $D$ and with a radius of $d$.

As shown in Fig. 5, all the nodes that are sending CB packets are located within the area $\mathcal{T}_{i}\left(\mathcal{T}_{i}=\mathcal{C}_{i}-\mathcal{S}_{D}\right)$.

Here we make the assumption that there is a common neighbor $m$ ' located in the intersection of $\mathcal{S}_{D}$ and $\mathcal{C}_{i}$.

According to the Greedy Forwarding used in this paper, $n_{i-1}$ chooses $m$ ' as the next hop rather than the node $n_{i}$, as the Euclidean distance between $D$ and $m$ ' is smaller than that between $D$ and $n_{i}$. This conflicts with the fact that $n_{i-1}$ and $n_{i}$ are two neighbor nodes located in the primary path. This confliction guarantees that neither node could be located in the intersection of $\mathcal{S}_{D}$ and $\mathcal{C}_{i}$. In other words, all the nodes that compete in sending $\mathrm{CB}$ packets are located within the area $\mathcal{T}_{i}\left(\mathcal{T}_{i}=\mathcal{C}_{i}-\mathcal{S}_{D}\right)$ only.

Two conditions should be satisfied when the number of nodes in $\mathcal{T}_{i}$ sending $\mathrm{CB}$ packets comes to a maximum: 1) the size of $\mathcal{T}_{i}$ comes to a maximum; 2) transmission area for each node sending $\mathrm{CB}$ packet has a minimal intersection with $\mathcal{T}_{i}$.

Condition 1. Both the size of $\mathcal{T}_{i}, \operatorname{Dis}\left(n_{i-1}, n_{i}\right)$ and $\operatorname{Dis}\left(n_{i}, D\right)$ have particular relationship with $\mu$. As shown in Fig. 5, we can calculate the size of $\mathcal{T}_{i}$ by (9).

$$
\begin{aligned}
\mathcal{T}_{i}= & 4 \int_{\frac{\mu R}{2}}^{R} \sqrt{R^{2}-x^{2}} d x-2 \int_{h}^{d} \sqrt{d^{2}-x^{2}} d x-2 \int_{R-h}^{R} \sqrt{R^{2}-x^{2}} d x \\
= & 2 R^{2} \arccos \frac{\mu}{2}-\mu R^{2} \sqrt{1-\left(\frac{\mu}{2}\right)^{2}}-\left(\pi-2 \arccos \frac{1-\mu}{2}\right) R^{2} . \\
& +(1-\mu) R^{2} \sqrt{1-\left(\frac{1-\mu}{2}\right)^{2}}-(1-\mu)^{2} R^{2} \arccos \frac{1-\mu}{2}
\end{aligned}
$$


Where $d=(1-\mu) R, \quad h=(1-\mu)^{2} R / 2$. According to the equation above, the size of $\mathcal{T}_{i}$ comes to a maximum when $\mu \approx 0.091$, which is calculated through Matlab.

Condition 2. As shown in Fig. 6, the transmission area has the minimal intersection with $\mathcal{T}_{i}$ when the CB packet sender is located at the boundary of $\mathcal{T}_{i}$, as $\overparen{A B}, \overparen{B C E}$ and $\overparen{E F}$ in the figure. Obviously, the closer those two $\mathrm{CB}$ packet senders (which are located at the boundary of $\mathcal{T}_{i}$ ) are located, the larger the number of CB packet senders is. The number reaches the maximum when $\operatorname{Dis}\left(m_{j}, m_{j+1}\right)$ is $R$. Therefore, we assume that the minimal distance between CB packet senders located at boundary of $\mathcal{T}_{i}$ is $R$.

According to Condition 2, we can calculate the number of $\mathrm{CB}$ packet senders in two ways: 1) the calculation begins at point $A$ in the anti-clockwise direction and at point $F$ in the clockwise direction simultaneously along the boundary of $\mathcal{T}_{i}$; 2) start in the anti-clockwise direction from the point $A$ and count orderly. Proof of the second way is similar to the first one, therefore only the first proof is given.

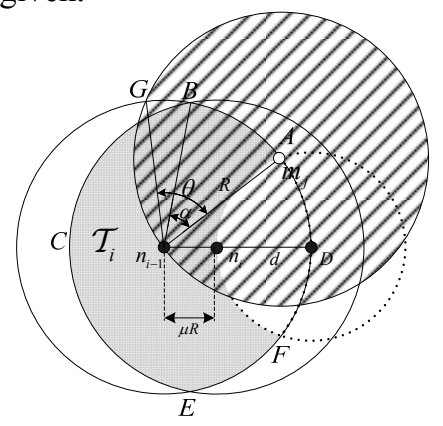

Figure 6. Radio coverage area for $m_{j}$.

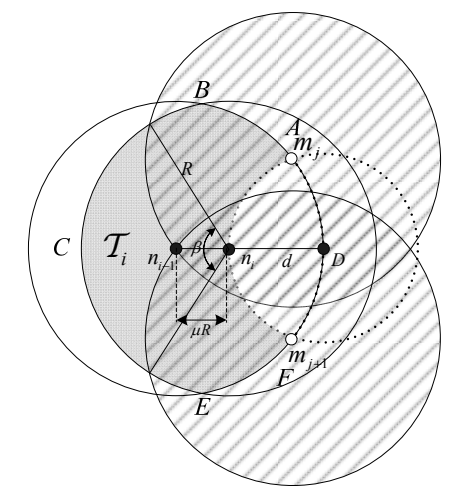

Figure 7. Radio coverage area for $m_{j}, m_{j+1}$.

In Fig. 6 and Fig. 7, $m_{j}$, and $m_{j+1}$ are two CB senders, $m_{j}$ is located at $A$ while $m_{j+1}$ at $F$, the slashed area denotes each node's transmission region respectively, $\mathcal{S}_{m_{j}}$ and $\mathcal{S}_{m_{j+1}}$ represent the transmission ranges of $m_{j}$ and $m_{j+1}$ separately.

As shown in Fig. 6, the effective coverage angle $\theta$ is the central angle produced by $m_{j}$ in the circle area centered at $n_{i-1}$, it's easy to get that $\theta=\pi / 3$ by geometry. $\theta$ denotes the effective area covered by $\mathrm{CB}$ packet sender in the area $\mathcal{T}_{i}$.

In geometry domain, $B$ and $E$ denote two points of intersection of the circle centered at $n_{i-1}$ and the circle centered at $n_{i} . \alpha$ is the central angle corresponding to $\overparen{A B}$ in the circle centered at $n_{i-1}$, noting that

$$
\alpha=\arccos \frac{\mu}{2}+2 \arccos \frac{1-\mu}{2}-\pi .
$$

When $\mu=0.091$ and $\alpha \approx 0.58$, we could get $\alpha<\theta$, which indicates that $\overparen{A B}$ and $\overparen{B C E}$ are in the area of $\mathcal{S}_{m_{j}}$. For the same reason, $\mathcal{S}_{m_{j+1}}$ covers the boundary of $\overparen{E F}$ and $\widehat{B C E}$.

In Fig. 7, $\beta$ is the central angle produced by area $\mathcal{T}_{i}-\mathcal{S}_{m_{j}}-\mathcal{S}_{m_{j+1}}$ in the circle centered at $n_{i}$, and could be calculated easily as

$$
\beta=\pi+\arccos \frac{1-\mu}{2}-2 \arccos \frac{(1-\mu) \sqrt{1+\mu}}{2} .
$$

When $\mu=0.091$, we could make the upper bound $\lceil\beta / \theta\rceil=2$, which indicates there are two nodes who can send CB packets in the area $\mathcal{T}_{i}-\mathcal{S}_{m_{j}}-\mathcal{S}_{m_{j+1}}$.

From analyses above, the number of nodes that are sending CB packets is no more than 4 during each link's local backup path setup.

\section{Performance Evaluation}

We evaluate the performances of LBR through OPNET10.0 and compare it with the high-reliability single-path routing protocol HARP (the third scheme of HARP in [5]), which sets up a route with a long lifetime by utilizing node's direction angle. Through experiment results, the performance evaluation is drawn.

\section{A. Evaluation Methodology}

In simulation, 200 mobile nodes are randomly and uniformly distributed in a $2000 \mathrm{~m} \times 2000 \mathrm{~m}$ rectangular area. The MAC layer protocol is based on IEEE standard 802.11 DCF (Distributed Coordination Function) [17], in which the channel bandwidth is $2 \mathrm{Mbps}$ and the radio transmission range is $250 \mathrm{~m}$.

The traffic flow uses CBR (Constant Bit Rate) with a data packet size of 512 Bytes. Each source transmitted data packets at a rate of 2 packets per second. The source and the destination of each CBR flow were randomly selected but not identical. Each flow did not change its source and destination throughout the simulation. The mobility model is based on random waypoint model with a speed in $\left[V_{\min } \mathrm{m} / \mathrm{s}, V_{\max } \mathrm{m} / \mathrm{s}\right]$ and a pause time of 0 second. In order to prevent nodes from degenerating to static behavior [18], we set $V_{\text {min }}=1 \mathrm{~m} / \mathrm{s}$. Each simulation lasts for 600 seconds and data in all figures is averaged over 30 runs.

We conducted two sets of simulations to evaluate the performance of LBR. First, we studied the performance of LBR with varying mobility velocity. Second, we 
studied the performance of LBR with varying number of CBR flows.

In order to evaluate the performance of LBR, we consider the following metrics:

(i) Packet delivery ratio: The ratio of the number of the successful arrived packets to the number of all packets transmitted by source. The larger this value, the better the protocol performance, which indicates that the more data packets are successfully delivered to destination.

(ii) End-to-end delay: The average time spent on data packet transmission from source to destination, including all possible types of delay during the transmission.

(iii) Route lifetime: The average duration from route establishment to route failure.

(iv) Control overhead: The total number of control packets per second. For HARP, control overhead comprises RREQ, RREP, RERR and the periodical beacon; while for LBR, there is no periodical beacon, and it includes RREQ, RREP, RERR and $\mathrm{CB}$. This metric indicates the routing cost.

\section{B. Impact of Varing Node Mobility Velocity}

In this set of simulations, we intend to evaluate the performance of protocols with the increase of node velocity. The number of CBR flows is fixed to 5 and $V_{\max }$ varies from 5 to $25 \mathrm{~m} / \mathrm{s}$

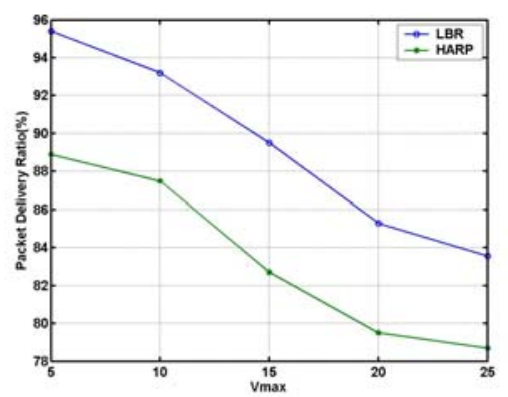

Figure 8. Packet delivery ratio vs. $V_{\max }$.

Fig. 8 shows the packet delivery ratio vs. $V_{\max }$. As we can see, with the increase of $V_{\max }$, the packet delivery ratios of these two protocols decrease. That is because path fails easily as the maximum node velocity increases. Furthermore, a data packet is dropped as the corresponding path fails, causing a reduction in packet delivery ratio. However, the packet delivery ratio of LBR is obviously higher than that of HARP. For example, when $V_{\max }$ reaches $25 \mathrm{~m} / \mathrm{s}$, the packet delivery ratio is still above $83 \%$. That is because HARP is a single-path routing protocol, path failure often occurs due to node mobility, and the data packets are dropped upon path failure. For LBR, data is continued through the corresponding local backup path if the primary path fails. That is, data packets are dropped only if both the primary path and the corresponding local backup path fail, which reduces the number of dropped data packets. LBR is more stable and has a good tolerance of varying node velocity.

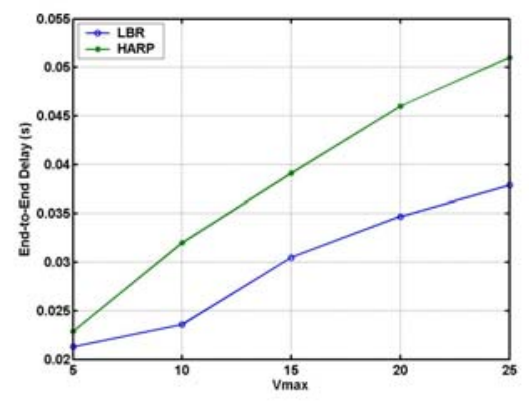

Figure $9 . \quad$ End-to-end delay vs. $V_{\max }$.

Fig. 9 shows the end-to-end delays vs. $V_{\max }$. It can be concluded that the end-to-end delay increases as $V_{\max }$ increases for two protocols. That is because as node velocity increases, topology changes faster, which more easily leads to link failure, route rediscovery, and an increase in transmission delay. However, the two routing protocols show different performances. LBR increases slowly, which indicates there is an improvement in transmission delay as node velocity increases. In LBR, when a link fails, no route rediscovery is needed for LBR and data transmission is continued through the local backup path; thus the end-to-end delay is reduced. However for HARP, route rediscovery is needed on path failure and the procedure of route discovery is timeconsuming; hence its end-to-end delay is higher than that of LBR.

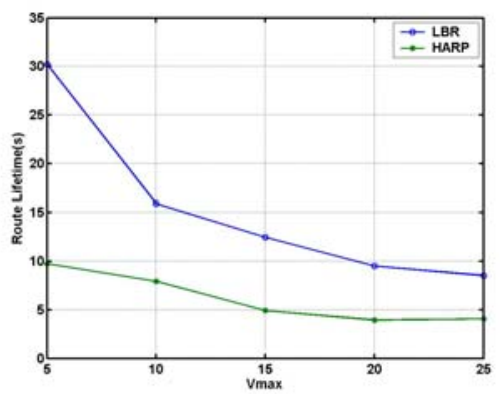

Figure 10. Route lifetime vs. $V_{\max }$.

Fig. 10 shows the route lifetime vs. $V_{\max }$. We can see that the route lifetime decreases when $V_{\max }$ increases for both protocols. When the node velocity increases, the stability of each link decreases. The main reason is that the increase of node velocity leads to an increase in the probability of link failure. However, the route lifetime of LBR is obviously higher than that of HARP. That is because local backup path is used in LBR, which increases route robustness and route lifetime. Therefore, the existence of a backup route can prolong route lifetimes and increase route stability. Hence, we can see that node mobility is a crucial factor in the route lifetime. 


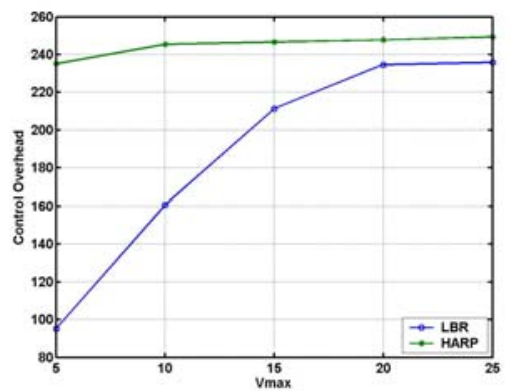

Figure 11. Control overhead vs. $V_{\max }$.

Fig. 11 shows the control overhead vs. $V_{\max }$. We can see that as node velocity increases, the control overhead of both protocols has an increasing tendency. As the node velocity increases, the frequency of link failure increases, and thus the control overhead is increased due to route rediscovery. However, when the node velocity is relatively low, LBR has a lighter control overhead than that of HARP, as there is no need of periodical HELLO packets, while HELLO packets are needed for HARP to obtain location information about neighbor nodes. When the node velocity increases, the control overhead of LBR increases fast. That is because RREQ is flooded to destination in LBR, and route rediscovery becomes more frequent, thus the number of RREQ packets and control overhead increases quickly correspondingly.

\section{Impact of Varing Number of CBR Flows}

In this set of simulations, we intend to evaluate the performance of protocols with different network traffic load. The maximum node velocity $V_{\max }$ is fixed to $10 \mathrm{~m} / \mathrm{s}$, while the number of CBR flows varies from 1 to 25 .

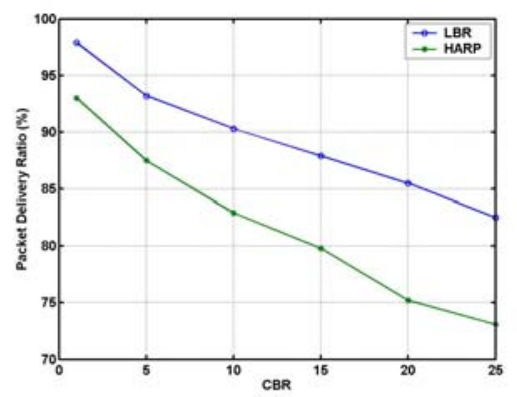

Figure 12. Packet delivery ratio vs. number of CBR flows.

From Fig. 12, the packet delivery ratio shows a decreasing tendency as the number of CBR flows increases for both LBR and HARP. However, the packet delivery ratio of LBR is higher than HARP. The control overhead increases as the number of CBR flows increases, thus the network becomes more congested and data packets are dropped, which leads to a decreased packet delivery ratio. LBR has a higher packet delivery ratio than that of HARP and shows a slower decreasing tendency. That is because route lifetime is prolonged through local backup paths in LBR, which relieves congestion and reduces the number of dropped packets.

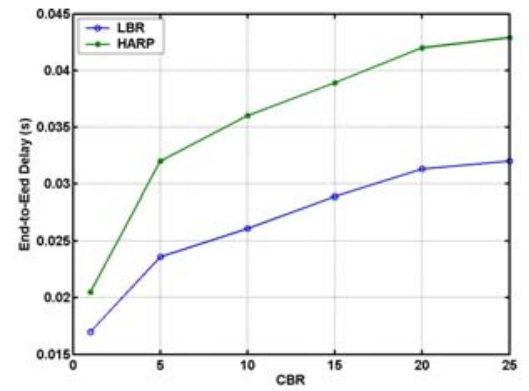

Figure 13. End-to-end delay vs. number of CBR flows.

Fig. 13 shows the end-to-end delay vs. the number of CBR flows. As the number of CBR flows increases, transmission delay is increased. That is because network overhead is increased as the number of CBR flows increases, and the network becomes more congested, which leads to an increased end-to-end delay. However, the delay of LBR increases relatively slower, as no route rediscovery is needed in case of primary path failure, and the local backup path is used instead, which reduces endto-end delay during the transmission and relieves network congestion.

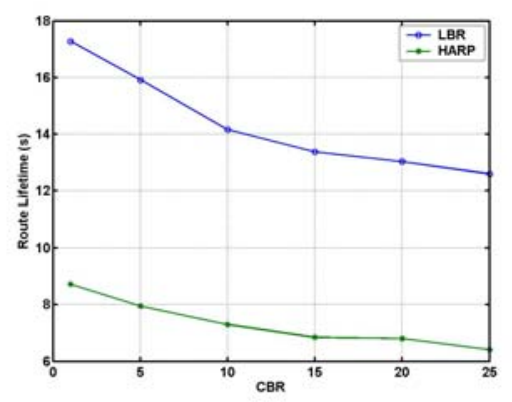

Figure 14. Route lifetime vs. number of CBR flows.

Fig. 14 shows the route lifetime vs. the number of CBR flows. As the number of CBR flows increases, the route lifetime changes slightly for both protocols. However, route lifetime of LBR is longer than that of HARP. Because the local backup path is used on primary path failure in LBR and a route rediscovery is necessary only when both the primary path and the corresponding local backup path fail. Hence route lifetime is greatly increased.

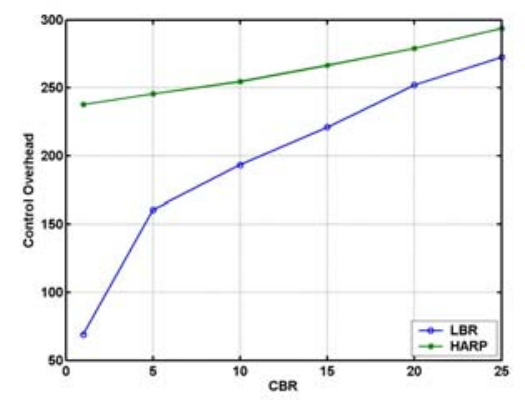

Figure 15. Control overhead vs. number of CBR flows. 
The impact of varying number of CBR flows on the number of control packets is shown in Fig. 15. As can be seen, the control overhead of two protocols has a growing tendency when the number of CBR flows increases. However, when the number of CBR flows is relatively small, the control overhead of LBR is lighter than that of HARP, since periodical HELLO packets are needed in HARP to obtain neighbor nodes' location information. As the number of CBR flow increases, the control overhead of LBR increases rapidly. That is because RREQ is flooded to destination for route discovery in LBR. As the number of CBR flow increases, the number of flooding RREQ packets increases and the control overhead is increased accordingly.

\section{CONCLUSION}

Dynamic topology and random mobility lead to instable route in MANETs. This paper proposes a novel MANETs routing protocol by using a link lifetime based multipath mechanism to improve route stability, which is called Link lifetime based Backup Routing protocol (LBR). During the local backup paths setup, LBR conduct a local optimization, which guarantees the backup path to have the maximum lifetime in local area. Further more, theoretical analyses demonstrate that the route lifetime is prolonged by the LBR backup scheme.

Extensive simulations are preformed to compare LBR with HARP. The simulation results show that LBR has good performances in terms of end to end delay, path lifetime and control overhead, etc. The main direction of our future research is to consider other issues on LBR such as Quality of Service (QoS).

\section{REFERENCES}

[1] Johnson DB, Maltz DA, and Hu YC. "The dynamic source routing protocol for mobile ad hoc networks (DSR)," IETF draft-ietf-manet-dsr-10.txt, 2004,07,19.

[2] Perkins CE, Belding-Royer EM, and Chakeres I. "Ad hoc on demand distance vector (AODV) Routing," IETF Internet draft draft-perkins-manet-aodvbis-00.txt, July 2003. RFC 3561.

[3] Nen Chung Wang, Jhu Chan Chen, "A Stable On-Demand Routing Protocol for Mobile Ad Hoc Networks with Weight-Based Strategy," Parallel and Distributed Computing, Applications and Technologies, 2006. PDCAT '06. Seventh International Conference on. pp.166-169, Dec. 2006.

[4] A. Nasipuri, R. Castaneda, S. R. Das. Performance of Multipath Routing for On-Demand Protocols in Mobile Ad Hoc Networks, Mobile Networks and Applications, vol.6, no.4, pp.339-349, 2001.

[5] Al-Akaidi M. and Alchaita M., "Link stability and mobility in ad hoc wireless networks," Communications, IET, vol.1, no.2, pp.173-178, April 2007.

[6] Brad Karp and PH. T. Kung, "GPSR: Greedy Perimeter Stateless Routing for Wireless Networks," 2000 Proceedings of the 6th annual international conference on Mobile computing and networking, pp.243-254, Aug. 2000.

[7] Song Guo, Yang O., and Yantai Shu, "Improving source routing reliability in mobile ad hoc networks," IEEE
Transactions on Parallel and Distributed Systems, vol. 16, no.4, pp.362-373, April 2005.

[8] Chun-Yen Hsu, Wu J.-L.C., Shun-Te Wang, "Finding stable routes in mobile ad hoc networks," 18th International Conference on Advanced Information Networking and Applications, 2004,pp.424-427,2004.

[9] Nen Chung Wang, Yung Fa Huang, JhuChan Chen, "A stable weight-based on-demand routing protocol for mobile ad hoc networks," Information Sciences, vol.177, pp.55225537, December 2007.

[10] Valera A.C., Seah W.K.G., and Rao S.V., "Improving protocol robustness in ad hoc networks through cooperative packet caching and shortest multipath routing," IEEE Transactions on Mobile Computing, vol.4, no.5, pp.443-457, Sept.-Oct. 2005.

[11] S.J. Lee and M. Gerla, "AODV-BR: Backup routing in Ad Hoc networks," Proceedings of IEEE WCNC 2000. Chicago, IL, (2000).

[12] Wei Kuang Lai, Sheng-Yu Hsiao, and Yuh-Chung Lin, "Adaptive backup routing for ad-hoc networks", Computer Communications archive, vol.30, no.2, pp.453-464, January 2007.

[13] J. Li, J. Jannotti, D. Couto, D. R. Karger, and R. Morris, "A scalable location service for geographic ad hoc routing," in Proceedings Sixth Annual ACM/IEEE International Conference on Mobile Computing and Networking (Mobicom), August 2000.

[14] Y. Ko, N. Vaidya, "Location-Aided Routing(LAR) in Mobile Ad Hoc Networks," Proceedings of the Fourth Annual ACM/IEEE International Conference on Mobile Computing and Networking (Mobicom '98), pp.66-75,1998.

[15] Su W., Lee S.-J., and Gerla M., "Mobility prediction in wireless networks," Proc. of IEEE MILCOM 2000, Los Angeles, CA, vol.1, pp.491-495, October 2000.

[16] S. Ross, Introduction to Probability Models (Academic Press, 1989).

[17] "Wireless LAN Medium Access Control (MAC) and Physical Layer (PHY) Specifications," IEEE Standard 802.11-1997, IEEE Standards Dept., 1994.

[18] J. Yoon, M. Liu, and B. Noble, "Random Waypoint Considered Harmful," Proc. Of IEEE INFOCOM, pp.1312-1321, 2003.

Wenjing Yang received the M.S. degree in computer science and technology from Xi' an Jiaotong University, China, in 2005. She is currently a Ph.D. candidate at the school of Electronic and Information Engineering from Xi'an Jiaotong University, China. She attends the Chinese 863 project "Research in heterogeneous mobile ad hoc networks". Her research interests include wireless communication and mobile ad hoc networks.

Xinyu Yang received the B.S., M.S. and Ph.D. degrees in computer science and technology from Xi'an Jiaotong University, China, in 1995, 1997, and 2001, respectively. He is now a full at the Department of Computer Science and Technology, Xi'an Jiaotong University. His research interests include network security, wireless communication and mobile ad hoc networks.

Shusen Yang received the B.S. and M.S. degree in computer science and technology from Xi' an Jiaotong University, China, in 2006 and 2008 respectively. He attends the Chinese 863 project "Research in heterogeneous mobile ad hoc networks". He is now a Ph.D. candidate in Imperial College London, UK. His current research interests include distributed systems and network optimization of wireless sensor networks.

Yage Gong received the M.S. degree in 2006. Her research interests include wireless communication and mobile ad hoc networks. 